\title{
An Integrated Information System for Financial Investment
}

\author{
Xiaotian $\mathrm{Zhu}^{1}$ and Hong Wang ${ }^{2}$ \\ 1 Old Dominion University, College of Business \& Public Administration, \\ Department of Finance, 2004 Constant Hall, Norfolk VA 23529 USA, \\ xxzhu@odu.edu \\ 2 North Carolina Agricultural and Technical State University, Department \\ of Business Administration, Greensboro, NC 27411, USA \\ hwang@ncat.edu
}

\begin{abstract}
With the globalization and integration of world financial markets, the success in information system has become a critical issue for financial investment institutes in financial service sector. Information system support has started to encompass the whole range of operational and decision-making activities in investment and financial industry. In this study, we take the challenge by integrating artificial intelligent techniques into the framework of the financial management information system. Particularly, we address the effectiveness of neural networks based trading strategy decision support system and discuss how it could be integrated into the information system to improve uncovering accurate trading signals and maximizing trading profits. In addition, we also analyze the investment firms' complicated and dynamic environment, where the sources of information for trading decision making comes from, and show the advantage of artificial intelligent techniques in dealing with such nonlinear and complex information. The results obtained from this study demonstrate the potential value of neural networks in financial management information systems, by discovering patterns and trading signals in noisy and dynamic financial data and by integrating with other decision support systems in making a more optimized trading strategy.
\end{abstract}

\section{Introduction}

Under the environment of globalization and integration of world financial markets, the success in information system has become a critical issue for financial investment institutes in financial industry. The diversity and complication of domain knowledge existing in modern financial market makes it very difficult for investors

Please use the following format when citing this chapter:

Zhu, X., Wang, H., 2006, in Intcrnational Fedcration for Information

Processing, Volume 205, Research and Practical Issucs of Enterprise Information Systems, eds.

Tjoa, A.M., Xu, L., Chaudhry, S., (Boston:Springer), pp.449-456. 
to make correct investment decisions efficiently, since transaction speeds have become much faster nowadays. Therefore, there is a great necessity for developing a set of financial management information system for supporting decision-making and implementing optimal trading strategies in financial investment $[1,2]$.

Financial investment is a knowledge-intensive industry. Under the fast development of information and electronic transaction technologies, large amount of financial transaction data and market information have been collected in the last decades and the emergence of knowledge discovery technology sheds light toward building up various financial investment decision support systems [3]. Data of financial investment markets are essentially noisy and dynamic time-series which bring more challenges than the traditional discrete data for uncovering the hidden knowledge for trading decisions [4]. It's the highly dynamic and risky nature of modern investment environment that calls for applying some new technologies in dealing with such noisy and non-linear data and information in financial markets. Artificial Intelligence technology, especially Artificial Neural Networks, a computing system containing many simple nonlinear computing unites or nodes interconnected by links, are well-tested method for financial analysis on the financial markets [9]. Therefore, for non-linear trading signal prediction and trading decision support system the key is that they include artificial intelligence techniques like neural network, fuzzy logic, genetic algorithm and so on $[5,6]$. In this research we integrate an artificial neural network based investment decision support system into the framework of enterprise financial management information system and show how it take the advantage of abstracting underlying nonlinear financial relationships for trading signal prediction and how it is integrated with other decision support and trading systems to enhance the accuracy and efficiency of the enterprise information system on the whole. By abstracting appropriate trading rules based on predicted trading signals and further reconfirmed with the results form other investment decision support and trading systems, neural network based decision support system could add value on the enterprise investment information system and enhance both its accuracy and efficiency on the whole.

The paper is organized as following: Section II analyzes the complex and dynamic financial environment for the financial investment institutes; Section III introduces the process of integrating the neural network based decision support system into the framework of enterprise financial investment information system; Section III provides some analysis on the integrated information system and draws the conclusions.

\section{Analysis of the Financial Environment}

With the development in advanced information technologies, modern financial market has became more and more dynamic and efficient in the sense of intra- and inter- market information exchange and transformation. As shown in Fig. 1, there are 
four main interconnected entities that make up the macro-architecture for the information system of the financial markets: Interlinked financial markets, market participants, public information, and government regulators. It is the information exchange and interactions based on the transferred information among these four interconnected entities under this framework that dynamically and continuously determine the market price and the tendency of the market index. Particularly, there are mainly six pairs of interacted relationships under this framework:

1) Financial Markets and Market Participants: It's through the market trading system that market participants directly (institute trader or investment broker/dealer) or indirectly (through broker/dealer) make transactions on the financial markets. The transactions ordering and quoting information update the financial market information system continuously and instantly reflected on the market board information and thus released as public information. On the other hand, based on both historical and live data from the financial markets, the market participants adjust their market expectations and market trend prediction for their future transactions.

2) Financial Markets and Public Information: continuously updated financial market board information, primary market news, secondary market movements and other macroeconomic or regulation news are the main sources for the public information. Reversely, released public information can affect financial market by changing investors' market expectations and by influencing the policy of market regulators.

3) Market Participants and Public Information: market participants can contribute to public information by their transactions on the first and secondary financial markets. On the other hand, the released public information can influence the market participants' market expectations, trend predictions and even trading strategies.

4) Market Participants and Government Regulator: government regulators can regulate the activities of market participants by limiting their over speculating and market making transactions to stabilize the financial markets. On the other hand, government regulators can also influence market participants' market expectations and transactions through monetary or fiscal policies. Reversely, market participants can influence the government regulator's policy by public information that reflecting the current market status and investors' expectations.

5) Government Regulator and Public Information: government regulators can directly influence the public information either by monetary policy from Central Bank or by fiscal policy from Treasury. The public information after releasing of these policies that reflecting both the macroeconomic status and market expectations can reflect the results of the government policies and thus influence the policy makers' decisions on future policy adjustment and regulations.

6) Government Regulator and Financial Markets: government regulators can influence financial markets either by regulating the market activities of speculators and arbitrageurs or by affecting market participants' expectations through monetary or fiscal policies. The financial market status resulting from the government regulations reversely can influence the government future decisions on policy making. 
From the above description, we can see that modern financial market is a highly dynamic and integrated information exchange and market price determining system. It was within such system that the financial price and trading volume information, market expectations, public and macroeconomic information and government regulations are continuously exchanged and interacted. It was under such dynamic mechanism the financial market system's functions are efficiently optimized.

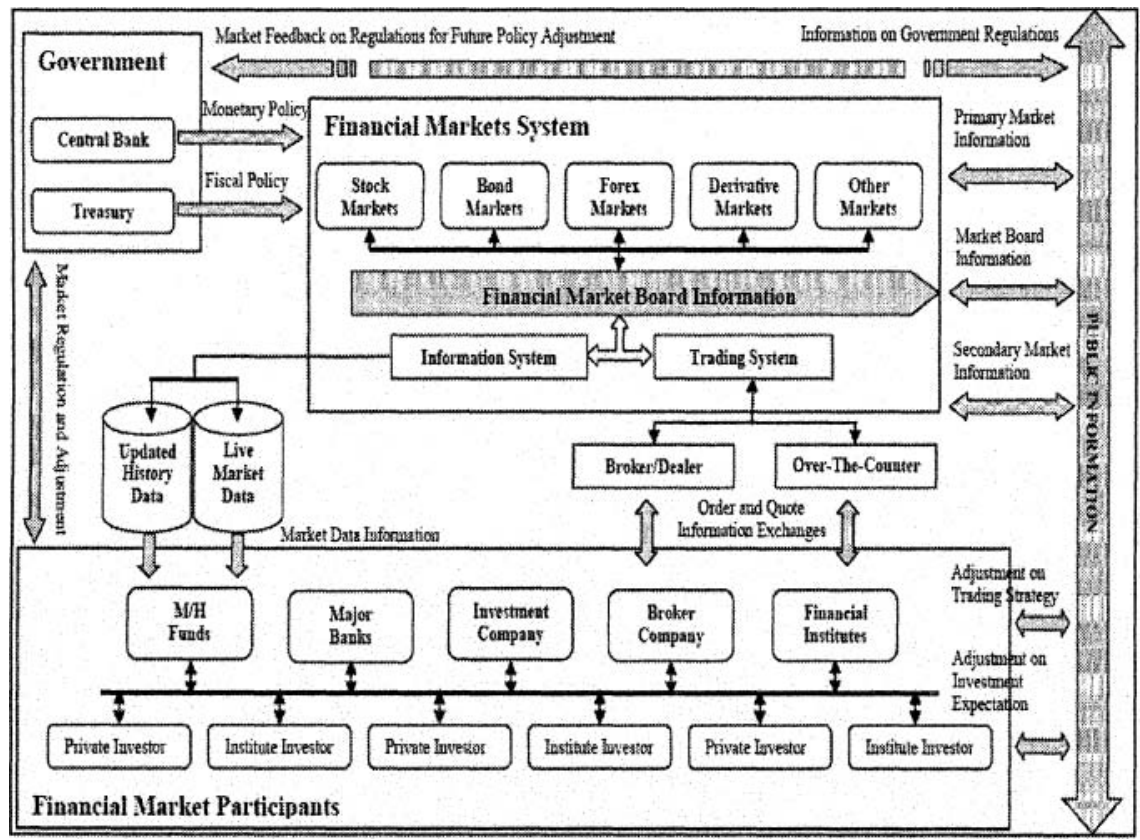

Fig. 1. Architecture of Information Systems in Financial Markets

Under such complex environment, the key to success, or even survival, for financial investment institutes is to integrate artificial intelligence techniques based investment decision support system into their enterprise investment information system. With machine learning, artificial neural network models the underlying nonlinear relationships among the entities of financial markets and allows appropriate learning, expression and presenting for better decision-making purposes [7].

\section{Integrated Enterprise Investment Information System}

There are number of approaches within the literatures which deal with applying artificial neural networks techniques to investment and trading decision support 
systems. Although there appears to be no formal segmentation of these different approaches, we classify them into four categories [8]:

1). Time Series Forecasting - predict future data points using historical data sets. Neural networks are used in the DSS to predict the base financial time series data (e.g. price, index and return) or financial indicators derived from the base data. Many trading signals are based on the predicted financial indicators that frequently used in technical analysis.

2). Pattern Recognition and Classification - attempts to classify observations into categories, generally by learning patterns in the base data or indicators. Applications involves the detection of patterns and segregation of predicted base data or indicators into 'buy', 'sell' or 'hold' categories of trading signals based on the trading strategies of the investors. For the fundamental analysis, neural networks can also be used in the DSS for financial distress and bankruptcy prediction as well as for credit rating.

3). Trading Strategy Optimization - based on the predicted financial price, indicators, recognized financial patterns and trading signals, neural networks can determine the optimal point at which to enter the transactions under the appropriate trading strategies.

4). Hybrid - this category was used to distinguish research which attempts to exploit the synergy effect by combining more than one of the above styles.

In this study, we would integrate all these features of artificial neural networks into the process of investment decisions support system and fully take the advantage of neural network in dealing with nonlinear financial information. In another words, we will use a hybrid neural networks based decision support system and further integrated this DSS with other traditional DSS in the whole process of operational and decision-making process of the Enterprise Investment Information System. The traditional decision support systems for financial investment is mainly based on such linear forecasting techniques like ARMA modeling, logit estimation MACD technique models and naive strategy. Fig. 2 provides architecture on how to integrate the artificial intelligence technology into the framework of enterprise financial investment information system. Specifically, neural networks can improve the performance of the information system in each of the following procedures:

Step 1: Predictions. The integrated information system in this procedure will use forecasting techniques to predict the future values of price or indicators. As mentioned above, neural networks have advantage comparing with other traditional forecasting techniques in recognizing the underlying nonlinear relationships in the complex financial information thus can make more accurate predictions on both future prices and indicators. In this procedure, both the neural network and traditional techniques based DSS will make their own predictions.

Step 2. Trading Rules. System in this procedure will generalize some trading rules and thus trading strategies based on the historical and predicted market value and indicators. Trading strategies are a way of implementing a set of mechanical trading rules to determine when to buy and sell a set of instruments. Trading strategies 
enable the investor to develop a systematic approach to trading, test how a system does in the past, and use signals from the system to place trades into the future. With machine learning, neural networks could learn the patterns in the financial price and indicators and generalize more reliable trading rules than traditional techniques [9] Step 3. Backtest and Trading Rules Optimization. System at this procedure would backtest and compares all the trading rules generalized from both neural networks and traditional techniques based DDS and choose the optimal trading rules for future processing. At the same time, recursive mechanisms would be applied to adjust the settings of neural networks and new trainings and predictions are conducted based on the adjustment.

Step 4. Trading Signals and Transactions. System at this procedure will generalize trading recommendations categorized into 'Buy', 'Sell' and 'Hold' to the human expert or traders of the investment institute. While, these automatically generalized recommended trading signals are based on the system generalized 'optimized' trading strategies, the system users or the traders can make decisions by their own human judgment based on the system forecasted indicators or patterns.

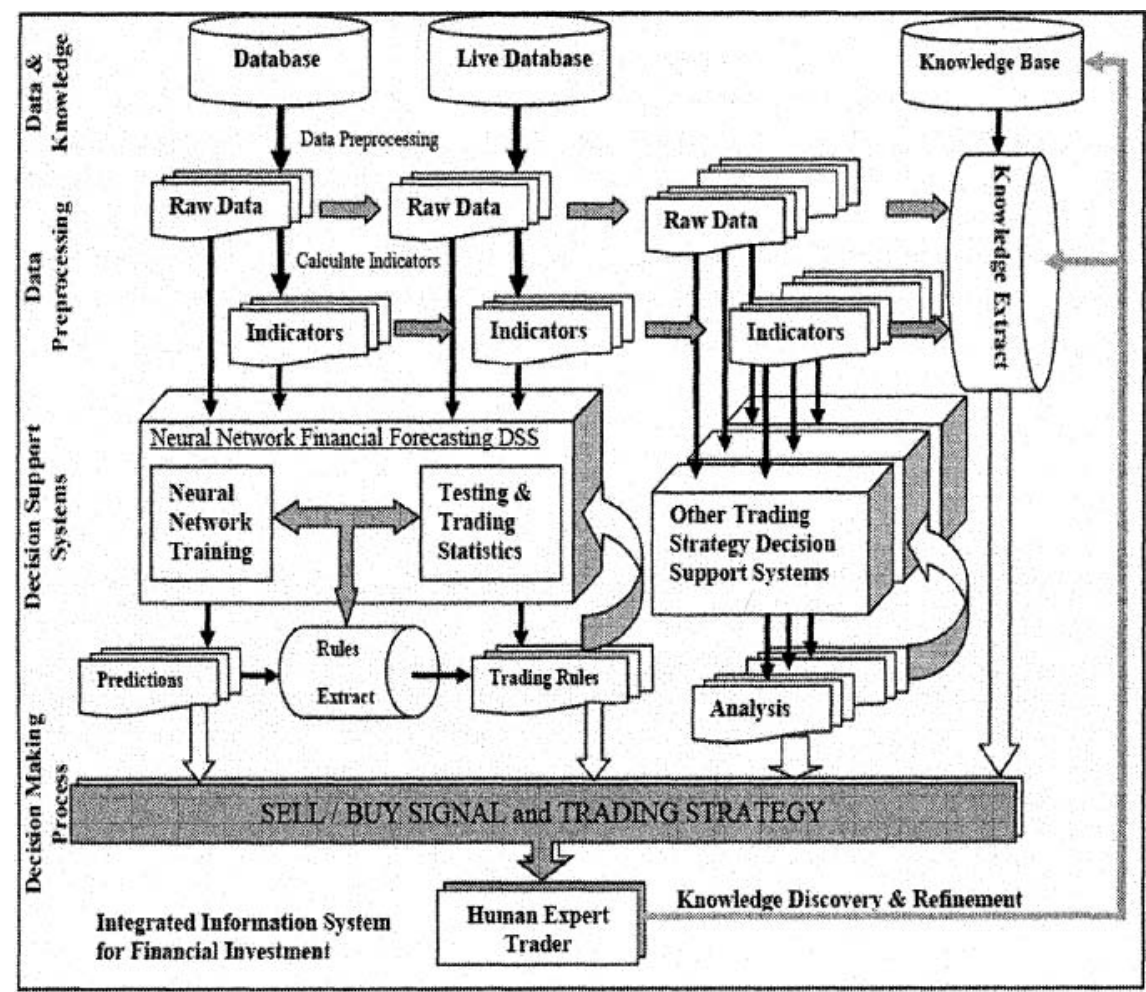

Fig. 2. Integrate Artificial Intelligence DDS into the Framework of Enterprise Financial Investment Information System 
Such a hybrid investment information system used will lead to investment results that could not have been obtained using only conventional techniques.

\section{Conclusions}

Under current complex and dynamic financial environment, efficient financial investment information system has became critical for the success or even survival of the investment institutes. Data of financial market is essentially noisy and nonlinear time-series which brings more challenges than the traditional discrete data for uncovering the hidden knowledge. In this study, in order to overcome the above challenges, we integrate the artificial intelligence technique based DSS into the framework of enterprise investment information system to improve its performance by discovering patterns and trading signals in noisy and dynamic financial data and by integrating with other decision support systems in making a more optimized trading strategy. In particular, among the results obtained we can mention the following:

1). By the detailed analysis on the inter-relationships among the main financial market entities, this study provided the financial investment institutes a dynamic and comprehensive architecture of the interconnected financial market environment.

2). This study provided a classification of neural network advantages in the aspect of investment decision support applications and further integrated these advantages step-by-step into the framework of enterprise investment information system.

The results obtained from this study demonstrate the potential value of neural networks in financial management information systems.

\section{References}

1. M.F. Jan, S. Andrew, and B. Whinston, A Web-Based Financial Trading System, IEEE 64$70(1999)$.

2. C. Zopounidis, C.M. Doumpos, and N.F. Matsatsinis, N. F., On the use of Knowledgebased Decision Support Systems in Financial Management: A Survey, Decision Support Systems 20, 259-277 (1997).

3. W. Leigh, N. Modani, R. Purvis, and T. Roberts, Stock Market Trading Rule Discovery Using Technical Charting Heuristics, Expert Systems with Applications 23, 155-159 (2002).

4. B.F. Irma, H.Z. Stelios, and W. Steven, Knowledge Discovery Techniques for Predicting Country Investment Risk, Computers and Industrial Engineering 43, 787-800 (2002).

5. T. Shin and I. Han, Optimal Signal Multi-resolution by Genetic Algorithms to Support Artificial Neural Networks for Exchange-Rate Forecasting, Expert Systems with Applications $18,257-269(2000)$. 
6. J. Van den Berg, U. Kaymak, and W. Van den Bergh, Financial Markets Analysis by Using a Probabilitistic Fuzzy Modeling Approach, International Journal of Approximate Reasoning 35, 291-305 (2004).

7. K. Shu-Ching, L. Sheng-Tun, C. Yi-Chung, and H. Men-Hsieu, Knowledge Discovery with SOM Networks in Financial Investment Strategy, In the Proceedings of the Fourth International Conference on Hybrid Intelligent Systems.

8. C.N.W. Tan, Artificial Neural Networks: Applications in Financial Distress Prediction and Foreign Exchange Trading, Gold Coast, QLD, Wilberto (2001).

9. A.P.N. Refenes, A.N. Burgess, and Y. Bentz, Neural Network in Financial Engineering: A Study in Methodology, IEEE Transactions on Neural Networks 8(6), 1222-1267 (1997). 